\title{
Tumor sólido seudopapilar del páncreas. Reporte de un caso.
}

\author{
Solid-pseudopapillary tumor of pancreas. A case report. \\ Edgar Núñez ${ }^{1}$, Carlos Zamalloa ${ }^{2}$, Vagner Frias ${ }^{3}$, Nancy Mayo ${ }^{4}$.
}

\section{RESUMEN}

El tumor sólido seudopapilar del páncreas es poco frecuente y de baja agresividad biológica. Afecta con mayor frecuencia a mujeres jóvenes y su pronóstico suele ser bueno después de la resección quirúrgica. Se presenta el caso de una mujer de 19 años, con tumor sólido pseudopapilar de páncreas, quien fue tratada mediante pancreatectomía distal con conservación esplénica.

PALABRAS CLAVE: Neoplasias pancreáticas, neoplasia, cirugía general (Fuente: DeCS BIREME)

\section{SUMMARY}

Solid pseudopapillary tumor of the pancreas is very rare, of low malignant potential and unknown origin. It generally occurs in young women and the prognosis is usually good after complete surgical removal. A female patient of 19 years is reported, with Solid-pseudopapillary tumor of the pancreas, who was treated by distal pancreatectomy with spleen preservation.

KEY WORDS: Pancreatic neoplasms, neoplasm, general surgery (Source: MeSH NLM)

\section{INTRODUCCIÓN}

Las lesiones quísticas del páncreas deben ser bien estudiadas dado que a diferencia de lo que ocurre en otros órganos, podrían corresponder a una neoplasia (1).

El tumor sólido seudopapilar del páncreas es poco frecuente, de baja agresividad biológica y de origen incierto, cuya línea celular aún no ha sido bien aclarada $(2,3,4)$. Es un tumor de células epiteliales primitivas con capacidad de diferenciación endocrina y exocrina y afecta con mayor frecuencia a mujeres jóvenes. La patogénesis del tumor no es conocida; sin embargo, la tendencia a afectar mujeres jóvenes ha sugerido que su origen puede tener relación con las hormonas sexuales $(5,6)$.

Se presentan las características clínicas, morfológicas, y el tratamiento efectuado en una paciente con diagnóstico de tumor seudopapilar del páncreas.

1 Cirujano Asistente, Servicio de Cirugía General. Hospital Nacional Cayetano Heredia. Profesor Principal. Departamento de Cirugía, Facultad de Medicina, Universidad Peruana Cayetano Heredia. Lima Perú.

2 Cirujano Asistente del Servicio de Cirugía General, Hospital Nacional Cayetano Heredia. Profesor Auxiliar. Departamento de Cirugía, Facultad de Medicina. Universidad Peruana Cayetano Heredia. Lima Perú.

3 Residente de Tercer año de Cirugía General, del Hospital Nacional Cayetano Heredia, Lima Perú.

4 Patólogo Asistente del Servicio de Patología del Hospital Nacional Cayetano Heredia, Lima Perú. 


\section{Caso clínico}

Mujer de 19 años sin antecedentes de importancia, acudió con historia de aproximadamente 7 años de malestar abdominal y dolor intermitente en el hipocondrio izquierdo y que se incrementaba con la deambulación. Concomitantemente notó tumoración abdominal que aumentó progresivamente de tamaño.

Al examen físico se encontraba en buen estado general y de hidratación, adolorida, colaboradora, ansiosa. No presentaba disnea ni ictericia. En abdomen se palpaba tumoración de $15 \times 15 \mathrm{~cm}$ en epigastrio, doloroso, móvil, no adherida a planos profundos y de bordes lisos.

Los análisis de laboratorio fueron normales y los marcadores tumorales y la prueba de Western Blot para quiste hidatídico, negativos. La radiografía de tórax fue normal, y en la tomografía se evidenció imagen ovoidea heterodensa proyectada anterior e inferior a la cola del páncreas, de $68 \times 108 \times 119 \mathrm{~mm}$ con tabiques internos tenues delgados y densidad interna entre $26 \mathrm{y}$ 45 UH con captación tenue del contraste; desplazaba las asas intestinales (Figura 1).

En el acto quirúrgico, se encontró una gran tumoración en la cola del páncreas de consistencia dura con adherencias al colon transverso. Se realizó pancreatectomia distal sin esplenectomía (Figura 2).

El informe de anatomía patológica fue: "Tumor encapsulado renitente, pardo oscuro, de $420 \mathrm{gr}$ y 10x9x8 cm. Al corte, presencia de múltiples cavidades quísticas de contenido sanguinolento, siendo la mayor de 3,5 cm de diámetro, con áreas amarillentas y necrosis. A la microscopía óptica: pared quística y septos fibrosos con amplias áreas de necrosis coagulativa. Presencia de áreas compactas de células
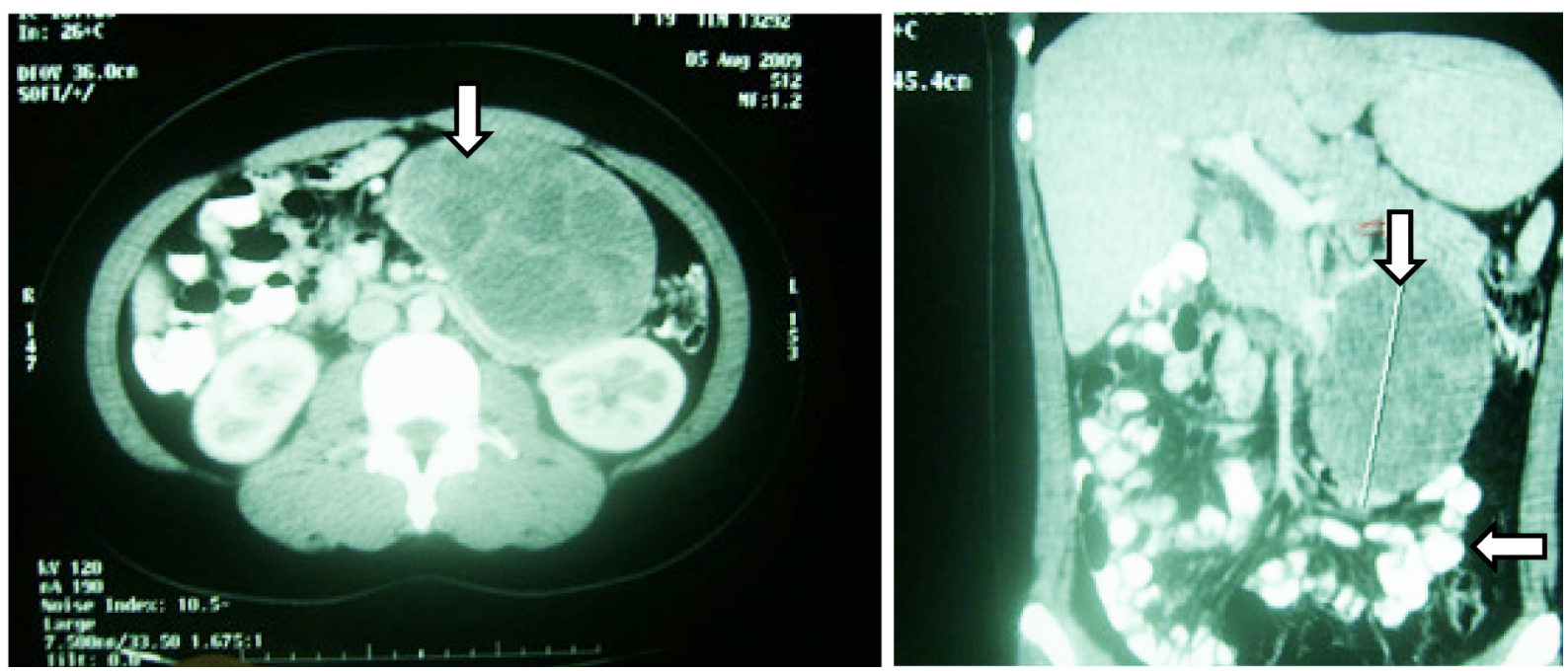

Figura 1. Tumoración multiquística de 11 x $12 \mathrm{~cm}$. Compromete la cola del páncreas y desplaza asas intestinales.
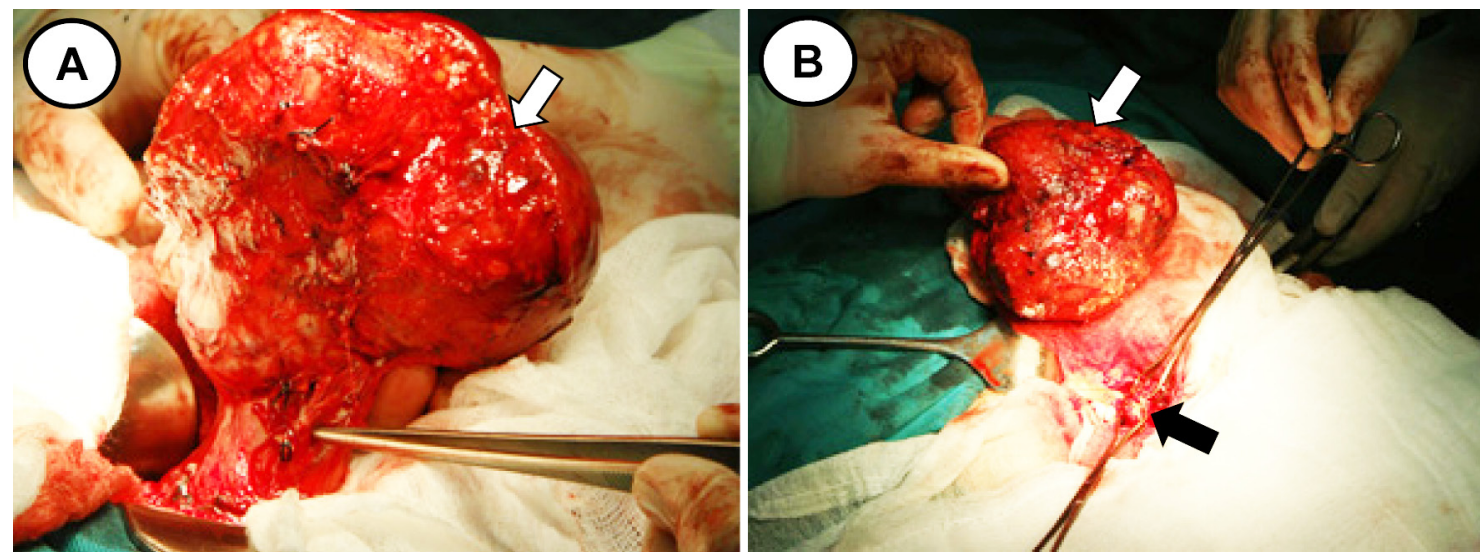

Figura 2. A: Tumor de cola del páncreas; B: pieza operatoria (flecha blanca) y exposición del muñón pancreático (flecha negra). 


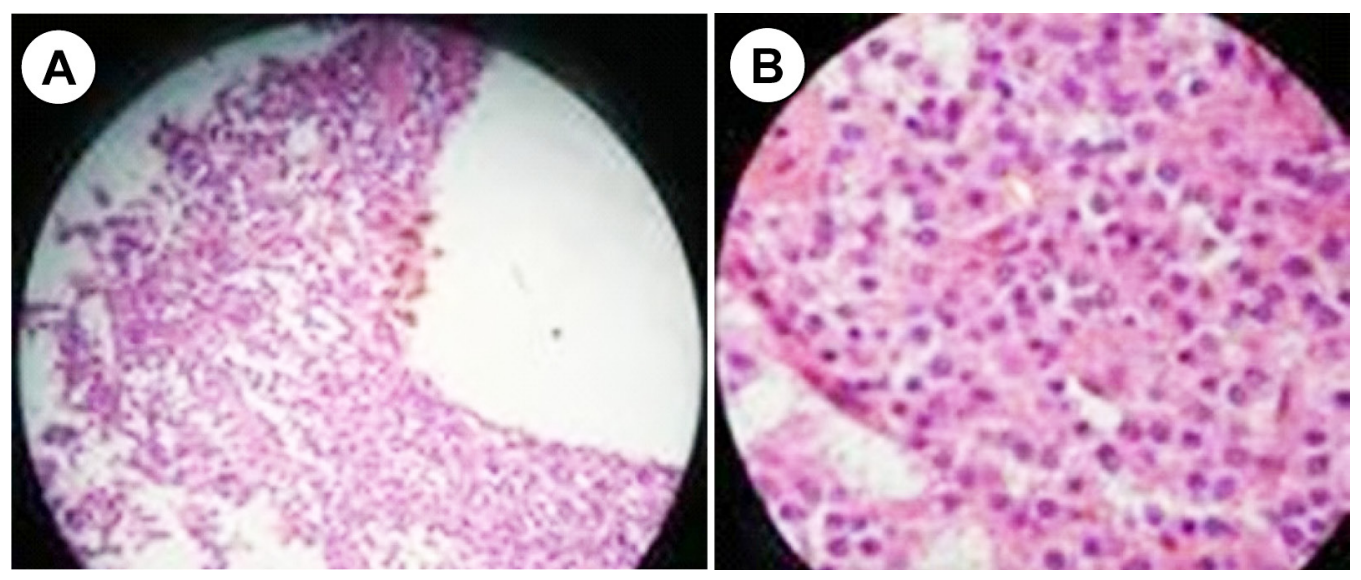

Figura 3. A: En el lado derecho de la imagen se observa área pseudo quística del tumor (HE 100X). B: Las áreas sólidas muestran sábanas de células epiteliales de núcleos redondeados con nucléolo rojo prominente (flecha) y citoplasma vacuolado eosinófilo no muy abundante. No se observa mitosis ni atipia nuclear (HE 400X).
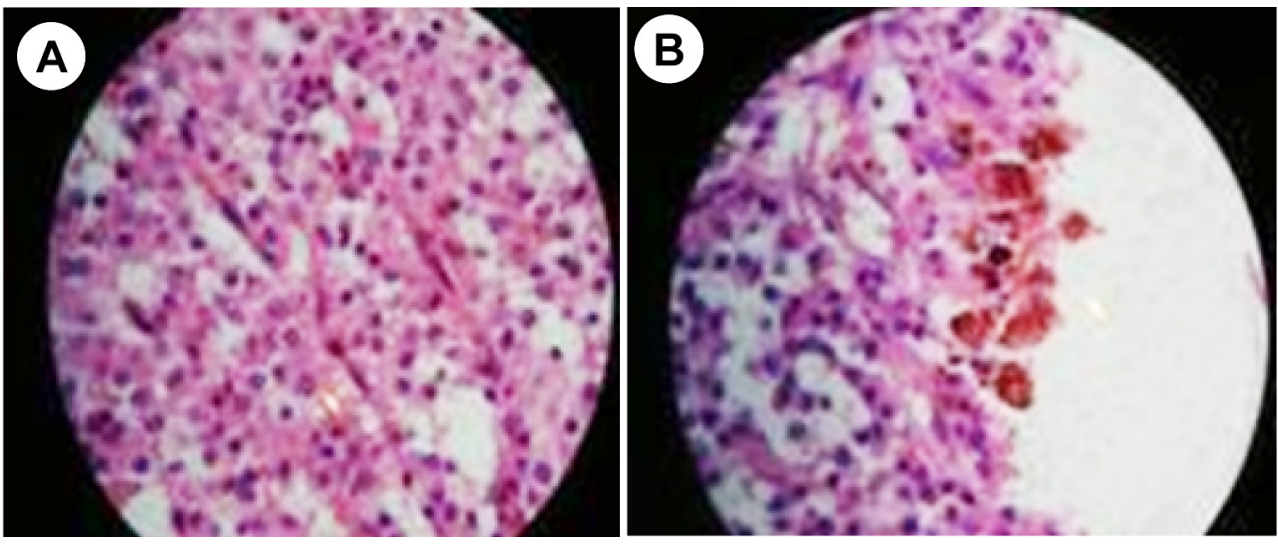

Figura 4. A: Las células monomorfas de pequeño tamaño, están separadas por finos capilares (HE 400X). B: Histiocitos xánticos fagocitando pigmento hemático (hemosiderina)(HE 400X).

monomorfas en tamaño, con patrón pseudopapilar, núcleos redondos con citoplasma eosinofílico de moderado tamaño, asociados a histiocitos xantínicos y otros fagocitando pigmento hemático. No se observó mitosis incrementada. Hallazgos compatibles con Tumor sólido pseudopapilar de páncreas." (Figuras 3 y 4).

La paciente permaneció hospitalizada 10 días, sin presentar complicaciones.

\section{DISCUSIÓN}

El cuadro clínico se caracteriza por malestar y dolor abdominal dependiendo del tiempo de evolución, producido por una tumoración abdominal que aumenta progresivamente de tamaño. No se asocia a enfermedades, ni a síndromes paraneoplásicos de secreción hormonal ni marcadores séricos específicos. Los análisis del laboratorio incluyendo los marcadores tumorales son normales.

El estudio morfológico es fundamental para decidir el tratamiento. El aspecto anatómico debe diferenciar un tumor, de un seudoquiste pancreático y además sugerir si es de naturaleza benigna o maligna. Por esto, el diagnóstico correcto es difícil antes de la operación y en la mayoría sólo el estudio histológico de la pieza quirúrgica nos da el diagnóstico definitivo.

El tumor generalmente es de tamaño considerable $(8-10 \mathrm{~cm})$ redondeado, con márgenes bien definidos pudiendo tener una apariencia sólida, sólida-quística o quística. La hemorragia intra-tumorales es frecuentes y también se ha descrito calcificaciones periféricas en el 30\% de los casos (6). El tratamiento habitualmente 
es quirúrgico y la resección completa del tumor es el tratamiento de elección, preservando la mayor cantidad de tejido pancreático que sea posible (7). En general el pronóstico es bueno.

El tumor sólido seudopapilar del páncreas fue descrito por Frantz (8), en 1959; pero recién en 1996, la OMS definió sus características y lo incluyó en la Clasificación Histológica Internacional de Tumores Pancreáticos Exocrinos. La denominación refleja correctamente las dos características histológicas encontradas con más frecuencia, las áreas sólidas y las pseudopapilares $(9,10)$.

Nuestra paciente era joven de sexo femenino, y la sintomatología de presentación fue la clásica con dolor abdominal producto de la tumoración pseudopapilar y del aumento progresivo de su tamaño, coincidiendo con lo descrito (11).

Dentro de las características morfológicas destacan el tamaño medio de $11 \mathrm{~cm}$, muy similar a la de nuestra paciente, ser unicéntricos e histología que varía en las diferentes regiones del tumor según su tiempo de evolución: las áreas quísticas y seudopapilares, resultan de cambios degenerativos $(12,13)$.

A pesar de poder alcanzar gran tamaño, el tumor seudopapilar del páncreas se clasifica dentro de las neoplasias de bajo potencial de malignidad y no suele dar metástasis; por esto, la escisión quirúrgica es mandatoria. Son de color pardo amarillento, de consistencia blanda y en cuya superficie de corte presenta áreas sólidas hemorrágicas, necróticas y quísticas, y compromete principalmente la cola de páncreas $(14,15)$.

El diagnóstico diferencial incluye, otros tumores quísticos como el cistoadenoma seroso, cistoadenoma mucinoso quístico y el pancreatoblastoma, entre otros. La inmunohistoquímica ayuda para diferenciar de tumores malignos (16), puede ayudar a diferenciar el tumor sólido seudopapilar de blastomas; sin embargo, la dificultad mayor es que no todos los hospitales cuentan con estas pruebas.

Es necesario tener siempre presente los criterios de malignidad: invasión pancreática y extrapancreática, metástasis a distancia, metástasis ganglionar e invasión vascular o perineural.

Dada la dificultad para diferenciar la naturaleza del tumor, es necesario realizar la cirugía en forma precoz.
El tipo de intervención dependerá de la naturaleza y de la ubicación del tumor. En nuestra paciente, se planteó la pancreatectomía distal con preservación esplénica, al estar el tumor situado a la izquierda de la línea media. Esta cirugía se puede realizar mediante dos técnicas: La primera, descrita por Mallet-Guy en 1943, consiste en dividir los vasos del páncreas y del bazo con mucho cuidado llegando hasta el plano posterior del páncreas, donde se procede a identificar y ligar los pequeños y cortos vasos pancreáticos que son ramas de la vena y arteria esplénica (17). La segunda, descrita por Warshaw en 1988, consiste en ligar los vasos esplénicos, dejando el bazo irrigado por los vasos cortos y los gastroepiploicos izquierdos; la conservación esplénica mediante ambas técnicas es segura en cuanto a la viabilidad del bazo $(18,19)$.

En general, se debe tratar de conservar el bazo como técnica de elección en lesiones benignas del cuerpo y cola del páncreas. Varios estudios muestran que la esplenectomía se asocia a mayor morbilidad en comparación con la cirugía conservadora. Los pacientes sin bazo, tienen mayor riesgo de presentar infecciones graves, como sepsis postesplenectomía (20-23).

Tagaya et al en 2003, publicó las indicaciones para la pancreatectomía distal laparoscópica, alternativa que se debe tener presente en este tipo de pacientes $(24,25)$.

No se ha reportado recurrencia del tumor, cuando se efectúa la resección completa del tumor (26).

\section{Correspondencia:}

\section{Edgar Núñez Huerta}

Hospital Nacional Cayetano Heredia

Av. Honorio Delgado s/n. San Martín de Porres

Lima 31

Correo electrónico: edgar.nunez@upch.pe

\section{REFERENCIAS BIBLIOGRÁFICAS}

1. Mao C, Guvendi M, Domenico DR, et at. Papillary cystic and solid tumor of the pancreas: a pancreatic embryonic tumor? Studies of tree cases and cumulative review of the world's literature. Surgery. 1995; 118: 821-8.

2. Kissane JM. Pancreatoblastoma and solid and cystic papillary tumor: two tumor related to pancreatic ontogeny. Semin Diagn Pathol. 1994; 11:152-64.

3. Pettinato G, Di Vizio D, Manivel JC. Solid- 
peudopapillary tumor of the pancreas: a neoplasm with distinct and highly characteristic cytological features. Diagn Cytopathol. 2002; 27:325-34.

4. Kleeff J, Diener M, Zgraggen $\mathrm{K}$, et al. Distal pancreatectomy: Risk factores for surgical failure in 302 consecutive cases. Ann Surg. 2007; 245:573-582.

5. Targarona J, Poggi L, Garatea R, et al. Tumores Sólidos Pseudotumorales de Páncreas. Rev Gastroenterol Peru.. 2007:27;186-190

6. Kang CM, Kim K, Choi JS. Solid pseudo papillary tumor of the pancreas suggesting malignant potential. Pancreas. 2006; 32:276-278.

7. Santini D, Poli F, Lega S. Solid-pseudopapillar tumor of the pancreas: histopathologhy. JOP. 2006; 7:131136.

8. Frantz VK. Tumors of the páncreas. Atlas of the Tumor Pathology. Section VII. Fasc.27 and 28 Washington, DC: Armed Force Institute of Pahology; 1959. p. 3279.

9. Abad-Licham M, Sanchez-Lihon J, Celis-Zapata J. Tumor sólido pseudopapilar de páncreas en el Instituto Nacional de Enfermedades Neoplásicas. Rev Gastroenterol Peru. 2008; 28:356-361.

10. Aydiner F, Erninac H, Savas B, et al. Pseudopapillary tumor of the páncreas: Emphasis on differential diagnosis from aggressive tumors of the pancreas. Turk J Gastroente. 2006; 17:219-222.

11. Hao Z, Ting-Bo L, Wei-Lin W, et al. Diagnosis and treatment of solid-pseudopapillary tumor of the pancreas. Hepatobiliary Pancreat Dis Int. 2006; 5: 454-458.

12. Hsueh-Lien H, Shou-Chuan S, Wen-Hsiung C, et al. Solid-pseudopapillary tumor of the pancreas: clinical experience and literature review. World $\mathrm{J}$ Gastroenterol. 2005;11: 1403-1409.

13. Albores-Saavedra J, Simpson K, Bilello S. The clear cell variant of solid pseudopapillary tumor of the pancreas: A previously unrecognized pancreatic neoplasma Am J Surg Pathol. 2006; 30:1237-1242

14. Federle MP, Mc Grath. Cystic neoplasms of the pancreas. Gastroenterol Clinic of North America. 2007; 36:365-376.
15. Romics L, Oláh A, Belágyi T, Hajdú N, Gyürüs P, Ruszinkó V. Solid pseudopapillary neoplasm of the pancreas - proposed algorithms for diagnosis and surgical treatment. Langenbecks Arch Surg 2010:395(6):747-755.

16. Fernández J, Targarona J. Thayer S, et al. Incidental pancreatic cyst, clinicopathologic characteristic and comparison with sintomatic patients. Arch Surg. 2003; 138:427-434.

17. Mallet-Guy P, Vachon A. Pancreatitis Chronique gauches. Paris: Masson \& Cie; 1943.

18. Warshaw AL. Conservation of the spleen with distal pancreatectomy. Arch Surg. 1988; 123:550-3.

19. Aldridge MC, Williamson R. Distal pancreatectomy with and without splenectomy. Br J Surg. 1991; 78:976-9.

20. Carrere N, Abid S, Henri C, et al. Spleen-preserving Distal Pancreatectomy with Excision of Splenic Artery and Vein: A Case-matched Comparison with conventional distal pancreatectomy with splenectomy. World J Surg. 2007; 31:375-82.

21. Benoist S, Dugue L, Sauvanet A, et al. Is there a role of preservation of the spleen in distal pancreatectomy? J Am Coll Surg 1999; 188:255-60.

22. Shoup M, Brennan MF, McWhite K, et al. The value of splenic preservation with distal pancreatectomy. Arch Surg 2002; 137:164-8.

23. De Castro SM, Singhal D, Aronson DC. Management of solid -pseudopapillary neoplasms of the páncreas: a comparison with standard pancreatic neoplasm. World J Surg. 2007; 31:1129-1134.

24. Tagaya N, Kasama K, Suzuki N, et al. Laparoscopic resection of the pancreas and review of the literature. Surg Endosc. 2003; 17: 201-6.

25. Mabrut J, Fernández-Cruz L, Azagra JS, et al. Laparoscopic pancreatic resection: result of a multicenter European study of 127 patients. Surgery.

Recibido: 09/03/11

Aceptado para publicación: 13/12/11 